\title{
Cloning and Characterisation of Chlorophyll Synthase from Avena sativa
}

\author{
Heidi C. Schmid, Ulrike Oster, Julia Kögel, \\ Stefan Lenz and Wolfhart Rüdiger*
}

Botanisches Institut der Ludwig-Maximilians-Universität München, Menzingerstr. 67, D-80638 München, Germany

${ }^{\star}$ Corresponding author

The chlorophyll synthase gene from oat (Avena sativa) was cloned and expressed in Escherichia coli. The deduced amino acid sequence consists of $\mathbf{3 7 8}$ amino acids including a presequence of 46 amino acids. Deletion mutants show that a core protein comprising amino acid residues 88 to 377 is enzymatically active. The sequence of the mature protein shows $85 \%$ identity with the chlorophyll synthase of Arabidopsis thaliana and $62 \%$ identity with the chlorophyll synthase of Synechocystis PCC 6803. The gene is constitutively expressed as the same transcript level is found in dark-grown and in light-grown seedlings. The enzyme requires magnesium ions for activity; manganese ions can reconstitute only part of the activity. Diacetyl and N-phenylmaleimide (NPM) inhibit the enzyme activity. Site-directed mutagenesis reveals that, out of the 4 Arg residues present in the active core protein, Arg-91 and Arg-161 are essential for the activity. Five cysteine residues are present in the core protein, of which only Cys-109 is essential for the enzyme activity. Since the wild-type and all other Cys-mutants with the exception of the mutant C304A are inhibited by Nphenylmaleimide, we conclude that the inhibitor binds to a non-essential Cys residue to abolish activity. The role of the various Arg and Cys residues is discussed. Key words: Chlorophyll biosynthesis/Diacetyl/ $\mathrm{N}$-Phenylmaleimide/Prenylation reaction/Site-directed mutagenesis.

\section{Introduction}

The last step of chlorophyll (Chl) biosynthesis is the introduction of the tetraprenyl (phytyl or geranylgeranyl) side chain, catalyzed by the enzyme chlorophyll synthase. The activity of chlorophyll synthase was originally detected in etioplast membranes of Avena sativa L. (Rüdiger et al., 1980), and several investigations were performed with this plant material (Benz et al., 1980, 1981, 1983; Schoch et al., 1980; Benz and Rüdiger, 1981a, b; Lütz et al., 1981; Helfrich and Rüdiger, 1992; Helfrich et al., 1994). The enzyme showed a higher activity with geranylgeranyldiphosphate
(GGPP) than with phytyldiphosphate (PhyPP), and the free alcohol geranylgeraniol was also accepted when ATP was added. The enzyme of spinach chloroplasts was shown to be exclusively located in the thylakoid membrane (Soll et al., 1983). However, it was not clear whether only a single chlorophyll synthase was present in the etioplast membrane preparation and whether additional enzymes contributed to the observed activities.

Beyond the role of an enzyme, chlorophyll synthase has a regulatory or channeling function. This function was demonstrated by in vitro translation experiments with plastid preparations. The Chl-binding proteins ( $\mathrm{P} 700$, $\mathrm{CP} 43, \mathrm{CP} 47, \mathrm{D} 1)$ accumulate only if $\mathrm{Chl}$ is synthesized de novo via the chlorophyll synthase reaction in the translation mixture (Eichacker et al., 1990, 1992; Kim et al., 1994). No accumulation was observed if Chl itself was added to the translation mixture (L. Eichacker, personal communication). The most probable explanation is the direct transfer of $\mathrm{Chl}$ from chlorophyll synthase to the nascent peptide chains of $\mathrm{Chl}$ a-binding proteins resulting in their stabilization (Eichacker et al., 1996). It is also known that in the absence of Chl both nuclear and plastid encoded Chl-binding proteins do not accumulate but are proteolytically degraded (Bennett, 1981; Klein et al. 1988).

The activity and substrate specificity of chlorophyll synthase was subsequently demonstrated with the recombinant enzymes from Rhodobacter capsulatus, Synechocystis sp. PCC6803 (Oster et al., 1997) and Arabidopsis thaliana (Oster and Rüdiger, 1997). The reports indicated a resemblance of the Synechocystis enzyme with chlorophyll synthase from green plants and of the Arabidopsis enzyme with chlorophyll synthase of etiolated plants. However, the results obtained with prokaryotic and eukaryotic genes expressed in Escherichia coli cannot directly be compared with previous results obtained with membranes from spinach chloroplasts and from oat etioplasts. We describe here the cloning and heterologous expression of the Chl G gene from Avena sativa. The enzyme activity was compared with that of etioplast membranes from $A$. sativa. Furthermore, kinetic, in vitro mutagenesis and inhibitor studies allow drawing a first tentative model for the enzyme mechanism of chlorophyll synthase.

\section{Results}

Cloning of the Chlorophyll Synthase Gene from Avena sativa

The basis for the cloning of the chlorophyll synthase gene was a cDNA preparation from $4 \mathrm{~d}$ old etiolated oat seedlings. Conserved regions corresponding to amino 
acid residues $109-115$ and $358-366$ of the $\mathrm{G} 4$ sequence of $A$. thaliana (Gaubier et al., 1995) were selected for PCR amplification with suitable primers. With primers $\mathrm{F} 1$ and $\mathrm{R} 1$ a 754 bp fragment was obtained. This fragment was used for restriction with Bam HI yielding a 446 bp fragment which served for the preparation of a probe for Northern blot analysis (see below). Further primers were designed according to the sequence of the $754 \mathrm{bp}$ fragment. The remaining parts of the sequence were amplified with suitable primers (see Material and Methods) according to the RACE method (Frohmann et al., 1988; Rashtchian et al., 1992). The complete sequence (1428 bp) containing the coding region (bp 1-1137) was then amplified by PCR with sequence-specific primers. The sequence has been deposited in the EMBL database with the accession number AJ 277210. The deduced amino acid sequence of the cloned gene contains a typical transit sequence for import into plastids. According to the program ChloroP (Emanuelsson et al., 1999), the transit sequence includes amino acid residues 1-46. It contains the conserved dipeptide Met-Ala and a large number of Ser residues (10 out of 46; von Heijne et al., 1989), and the sequence around the putative cleavage site Val-Val-Cys $\downarrow$ Ala agrees with the semi-conserved motif (lle/Val)-X-(Ala/Cys) $\downarrow$ Ala (Gavel and von Heijne, 1990). Furthermore, three Arg residues are present in positions minus 7 to 9. Positive charges are found in the C-terminal part of many, but not all chloroplast transit sequences (von Heijne and Nishikawa, 1991; Keegstra and von Heijne, 1992). If not stated otherwise, the enzyme reactions described in the following sections were performed with the recombinant chlorophyll synthase including the presequence.

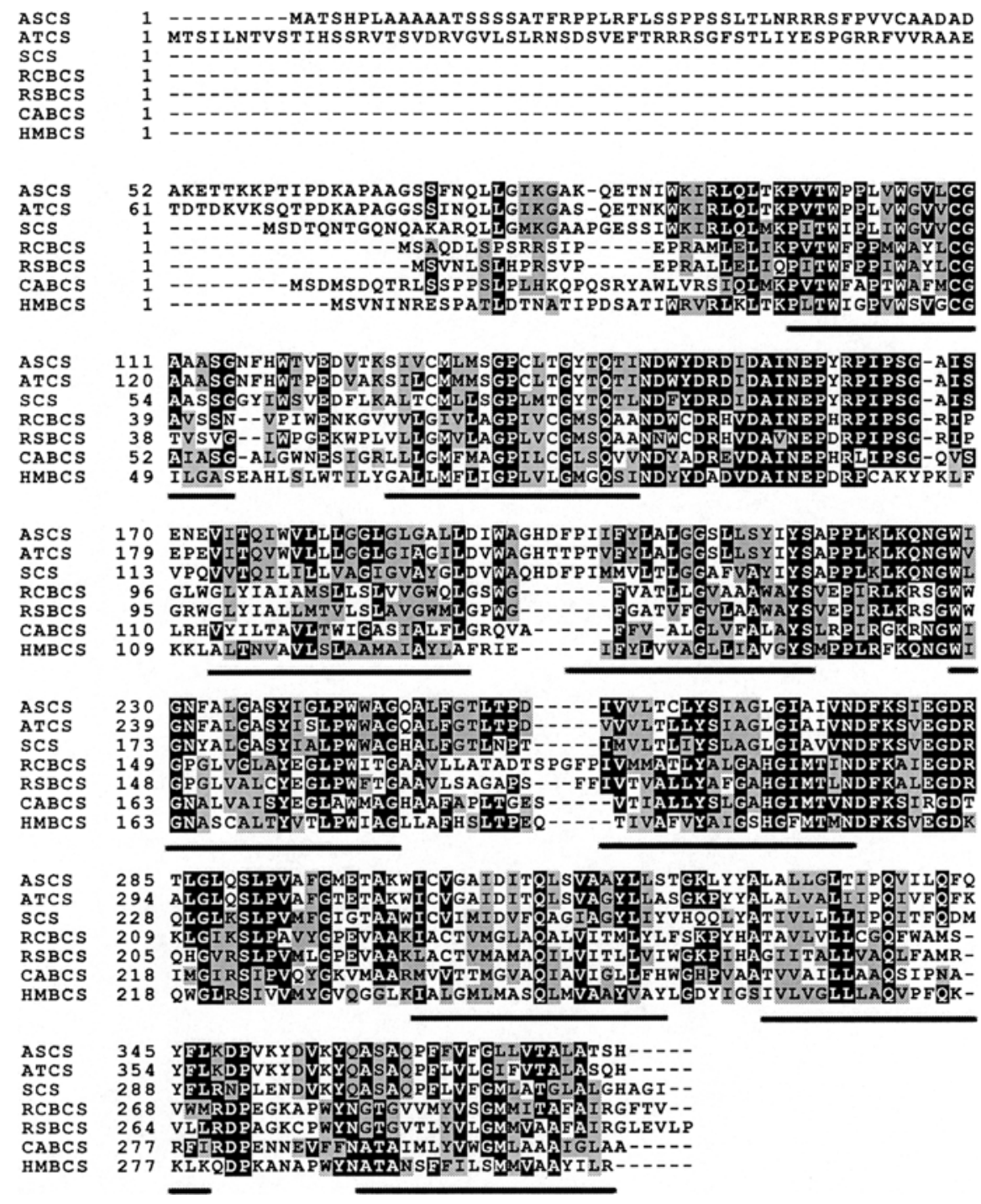

Fig. 1 Alignment of the Derived Amino Acid Sequence of $A$. sativa with Published Chlorophyll and Bacteriochlorophyll Synthase Sequences.

Identical residues are boxed in black, similar residues are in grey. Regions forming transmembrane helices predicted by the program HMMTOP are underlined.

Abbreviations: ASCS $=$ Avena sativa, ATCS $=$ Arabidopsis thaliana, SCS $=$ Synechocystis PCC6803, RCBCS $=$ Rhodobacter capsulatus, RSBCS $=$ Rhodobacter sphaeroides, CABCS $=$ Chloroflexus aurantiacus, HMBCS $=$ Heliobacillus mobilis . 
Table 1 Comparison of Chlorophyll Synthases from Avena sativa L. with the Known Chlorophyll Synthases, Expressed as \% Sequence Identity and Similarity, Respectively.

\begin{tabular}{|c|c|c|c|c|}
\hline \multirow[t]{2}{*}{ Plant material } & \multirow[t]{2}{*}{ Gene } & \multirow{2}{*}{$\begin{array}{l}\text { Mature protein } \\
\text { AA47-378 } \\
\text { Identity }\end{array}$} & \multicolumn{2}{|c|}{$\begin{array}{l}\text { Core protein } \\
\text { AA88-378 }\end{array}$} \\
\hline & & & Identity & Similarity \\
\hline Arabidopsis thaliana & G4 (chlG) & 85 & 87 & 93 \\
\hline Synechocystis PCC6803 & chlG & 62 & 67 & 80 \\
\hline Heliobacillus mobilis & bchG & 36 & 36 & 59 \\
\hline Chloroflexus aurantiacus & bchG & 36 & 43 & 67 \\
\hline Chloroflexus aurantiacus & bchG2 & 33 & 34 & 58 \\
\hline Rhodobacter capsulatus & bchG & 33 & 34 & 53 \\
\hline Rhodobacter sphaeroides & bchG & 31 & 32 & 54 \\
\hline
\end{tabular}

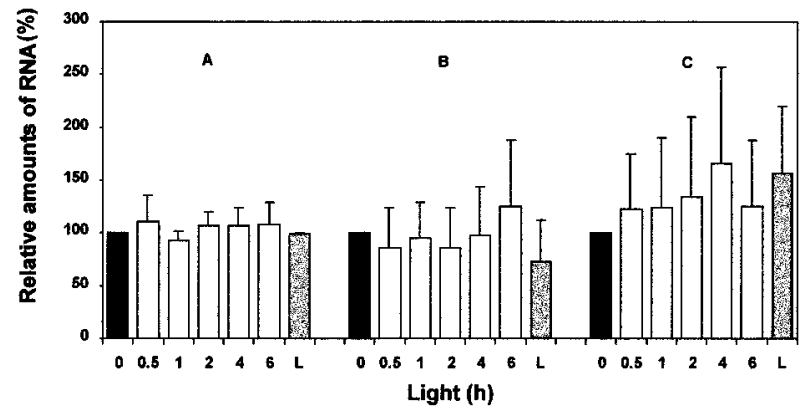

Fig. 2 Northern Blot Analysis of the Chlorophyll Synthase Transcript from 3 (A), 4 (B) and 5 (C) Day Old Oat Seedlings. The seedlings were grown in darkness and analyzed after exposure to white light for $0.5-6 \mathrm{~h}$ as indicated. The non-irradiated control is labeled $0 \mathrm{~h}$, and the label $\mathrm{L}$ refers to seedlings grown in continuous light for $3-5$ days. All values are based on the nonirradiated controls $(=100 \%)$.

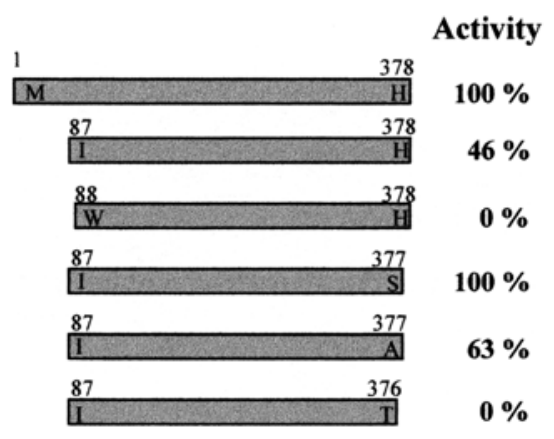

Fig. 3 Deletion Mutants of Chlorophyll Synthase and Their Enzymatic Activity.

The $\mathrm{N}$ - and $\mathrm{C}$-terminal residues of each fragment are given together with the percentage of enzyme activity in relation to the wild type.

The sequence of the putative mature protein (amino acid residues $47-378$ ) shows a high degree of identity to chlorophyll synthases from $A$. thaliana (85\%) and Synechocystis PCC 6803 (62\%), and to various bacteriochlorophyll synthases (31-36\%; Figure 1 and Table 1).
Prediction of the secondary structure using the program HMMTOP (http://www.enzim.hu/hmmtop.server/hmmtop.cgi) revealed 9 membrane-spanning helices for all enzymes as shown in Figure 1.

The same procedure was used to isolate a chlorophyll synthase gene from green plants. With a cDNA preparation from $4 \mathrm{~d}$ old light-grown barley seedlings, the obtained sequence proved to be identical with the sequence obtained from etiolated plants. These results agree with those of Gaubier et al. (1995) who found by Southern and Northern blot analysis that the chlorophyll synthase sequence represents a single-copy gene in $A$. thaliana. To test further whether different amounts of chlorophyll synthase can be detected in etiolated and green plants, Northern and dot blot analyses of etiolated greening and fully green oat seedlings were performed. As shown in Figure 2, the gene is constitutively expressed in etiolated and green oat seedlings, and no change in transcript levels is observable upon a shift from darkness to light (Figure 2).

\section{A Core Protein Is Enzymatically Active}

As expected, deletion of the presequence yielded a protein with full activity since this matched the sequence of the mature enzyme. Even further deletion of the N-terminus including amino acid residues 1-87 resulted in a 'core protein' that was still enzymatically active. However, the next residue, W88, was essential for activity: deletion of the $88 \mathrm{~N}$-terminal residues yielded a protein without enzymatic activity. At the C-terminus, only one residue $(\mathrm{H} 378)$ could be deleted without loss of activity, while deletion of S377 together with $\mathrm{H} 378$, and all shorter sequences showed no activity. Interestingly, the length of the sequence seems to be the crucial point since the mutant S377A was enzymatically active. The deletions are indicated in Figure 3.

\section{Metal Ion Requirement and Kinetics}

Most polyprenyl transferases require $\mathrm{Mg}^{2+}$ or other divalent ions for their activity. Here we tested this requirement 
Table 2 Metal lon Requirement of Chlorophyll Synthase from A. sativa.

\begin{tabular}{lr}
\hline Treatment & Chlorophyll synthase activity (\%) \\
\hline Without EDTA treatment & 100 \\
Pretreated with $100 \mathrm{~mm}$ EDTA & 2 \\
$4 \mathrm{mM} \mathrm{MgCl}_{2}$ & 95 \\
$4 \mathrm{mM} \mathrm{MnCl}_{2}$ & 31 \\
$4 \mathrm{mM} \mathrm{ZnCl}_{2}$ & 14 \\
$4 \mathrm{mM} \mathrm{CaCl}_{2}$ & 2 \\
\hline
\end{tabular}

Recombinant chlorophyll synthase from Avena sativa was incubated at $29^{\circ} \mathrm{C}$ with $50 \mathrm{~mm}$ HEPES-buffer ( $\mathrm{pH}$ 8.8) containing 4 $\mu \mathrm{M}$ Chlide and $350 \mu \mathrm{M}$ GGPP. The enzyme activity of the protein suspension without any further treatments refers to $100 \%$. Before the enzyme assay the protein suspension was treated with $100 \mathrm{~mm}$ EDTA for $30 \mathrm{~min}$. The EDTA was removed through three washing steps.

with the recombinant enzyme from Avena sativa. We did not find any reduction in the activity if $\mathrm{Mg}^{2+}$ was omitted from the reaction buffer and equimolar concentrations of EDTA were added instead. However, washing of the membrane preparation with a high concentration of EDTA reduced the activity of chlorophyll synthase dramatically (Table 2). The activity was restored with $\mathrm{Mg}^{2+}$, to a small extent also with $\mathrm{Mn}^{2+}$ or $\mathrm{Zn}^{2+}$, but not with $\mathrm{Ca}^{2+}$ (Table 2). We conclude that $\mathrm{Mg}^{2+}$ is essential for the reaction and that it is firmly bound to chlorophyll synthase so that high EDTA concentrations are required to remove the metal from the enzyme.

Etioplast membrane preparations accepted not only the diphosphates GGPP and PhyPP for the chlorophyll synthase reaction, but also the monophosphates GGMP and PhyMP and the non-phosphorylated alcohols geranylgeraniol and phytol, if ATP was present in the assay (Rüdiger et al., 1980). The recombinant enzyme did not accept the free alcohols even with a large excess of ATP in the assay. Therefore we conclude that etioplasts contain a specific kinase, which is not present in the bacterial lysate. However, the recombinant enzyme did catalyze the esterification of Chlide with GGMP, but the product yield was only $50-60 \%$ of the yield with GGPP irrespective of the presence or absence of ATP.

Upon separate incubation with the recombinant enzyme from $A$. sativa, GGPP reacted faster than PhyPP at the beginning (data not shown) in accordance with previous data obtained with the recombinant enzyme from Arabidopsis thaliana (Oster and Rüdiger, 1997). However, higher esterification was found with PhyPP than with GGPP upon prolonged incubation (data not shown). This became even more evident in the kinetics with a mixture of PhyPP and GGPP (Figure 4): while the absolute values for the pigment esterified with each of the alcohol moieties steadily increased, the percentage of $\mathrm{Chl}_{\mathrm{GG}}$ predominated at the beginning (1-15 $\mathrm{min})$ and the percentage of $\mathrm{Chl}_{\text {Phy }}$ predominated later (15-180 min). Control experiments revealed that the enzyme did not catalyse transesterification of $\mathrm{Chl}_{\mathrm{GG}}$ to $\mathrm{Chl}_{\mathrm{Phy}}$, thus the observa-

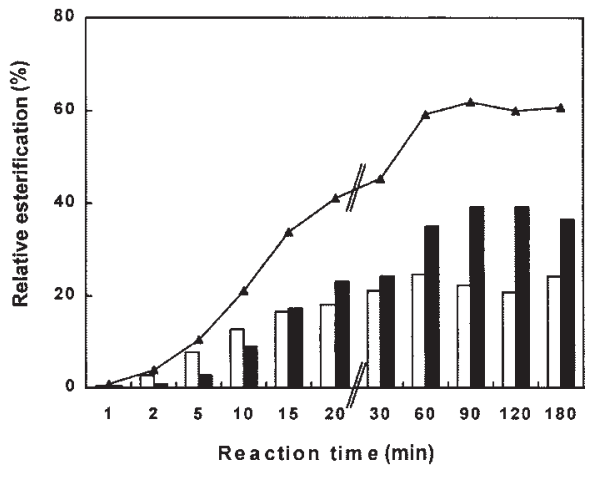

Fig. 4 Esterification of Chlorophyllide a with Geranylgeranyl Diphosphate (White Columns) and Phytyl Diphosphate (Black Columns).

The triangle indicates total esterification. Note the change in the scale of the reaction time.

tion must indicate a change of incorporation rates of the same enzyme for GGPP and PhyPP.

\section{Essential and Non-Essential Arginine and Cysteine Residues}

Specific inhibitors have often been used to identify essential amino acid residues of enzymes. Inactivation of liver prenyl transferase upon incubation with phenylglyoxal was interpreted by the presence of essential Arg residues (Barnard and Popjak, 1980). We tested the possible involvement of arginine and cysteine residues in the prenylation of Chlide with diacetyl and N-phenylmaleimide (NPM), respectively. The reaction conditions allowed esterification of 0.6-0.7 nmol Chlide with GGPP within the standard reaction time of $45 \mathrm{~min}$ (= control, $100 \%)$. First the enzyme of etioplast membranes was tested with the inhibitors. Significant inhibition was found after preincubation with $4 \mathrm{mM}$ NPM or $34 \mathrm{~mm}$ diacetyl (Figure 5A). The same results with the recombinant enzyme confirmed that the native and recombinant enzyme showed identical behaviour. The effects of the inhibitors were then investigated in more detail with the recombinant enzyme (Figure 5B). Inhibition could be observed much faster with diacetyl than with NPM. A plateau with $10-20 \%$ residual activity was reached after a preincubation of $30 \mathrm{~min}$. Longer preincubation resulted only in a very slow further decrease of activity. In subsequent experiments, the standard test for inhibition involved preincubation with the inhibitors for $30 \mathrm{~min}$.

Since the specificity of the inhibitors for certain amino acids can be questioned, a more convincing approach to detect essential amino acid residues makes use of sitedirected mutagenesis. This method con only be applied to the recombinant enzyme. The core protein (see Figure 3) contains 4 Arg and 5 Cys residues. We mutated each of these residues to Ala and tested the activity of the mutated enzyme without and with the corresponding inhibitor.

Two of the four Arg residues proved to be essential for chlorophyll synthase activity, as the mutants R91A and 
(A)
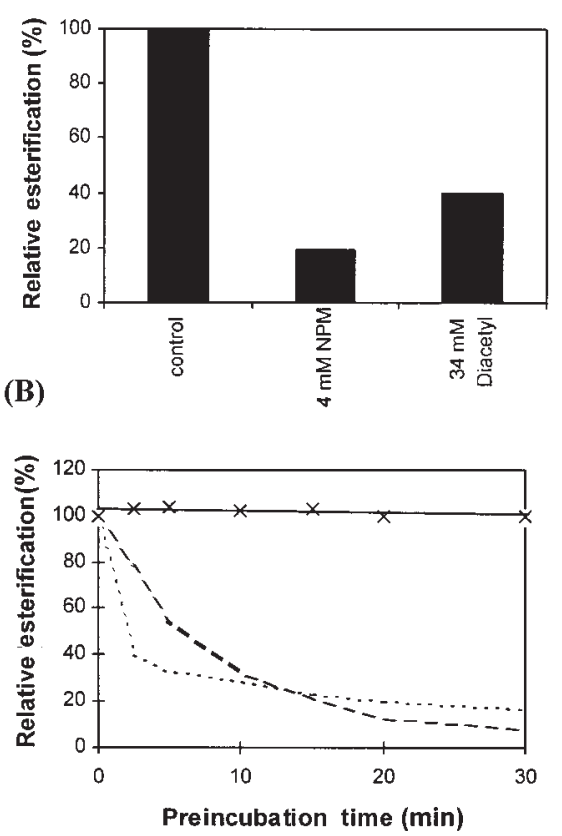

Fig. 5 Inhibition of Chlorophyll Synthase Activity by N-Phenylmaleimide (NPM) and Diacetyl.

(A) Etioplast membranes were preincubated with the inhibitors as indicated for $30 \mathrm{~min}$, and the enzyme activity was determined in comparison to the control preincubated with buffer. (B) The recombinant enzyme was preincubated for the indicated period of time with buffer (solid line), NPM (dashed line), and diacetyl (dotted line). The enzyme activity was determined at the time points indicated by crosses.

R161A did not retain any trace of activity. Some activity (about $25 \%$ of the wild type) was still found in the mutant R151A, and the mutant R284A exhibited wild-type activity (Figure 6A). Preincubation with diacetyl reduced the activity of the mutants R151A and R284A, indicating that either both or one of the residues not mutated in these experiments, R91 and/or R161, must be the essential point of attack of diacetyl.

Inhibition of enzymes by thiol-specific reagents has very often been taken as an evidence for the involvement of cysteine residues in the enzymatic reaction. However, such an assumption was not supported by subsequent experiments with chlorophyll synthase. Only one Cys residue of the 5 Cys residues present in the enzyme from A. sativa is conserved in all known chlorophyll synthase sequences (Figure 1). We found that the recombinant bacteriochlorophyll synthase from Chloroflexus aurantiacus, which contains the conserved Cys and only one additional Cys in the sequence (Figure 1), was not inhibited by NPM under the standard conditions (data not shown). Thus the inhibition of the enzyme from $A$. sativa must be due to the reaction of NPM with one or more of the other non-conserved Cys residues.

For a more detailed investigation of the NPM effect, point mutations were constructed for all Cys residues and the enzyme activity of the recombinant mutated en-
(A)

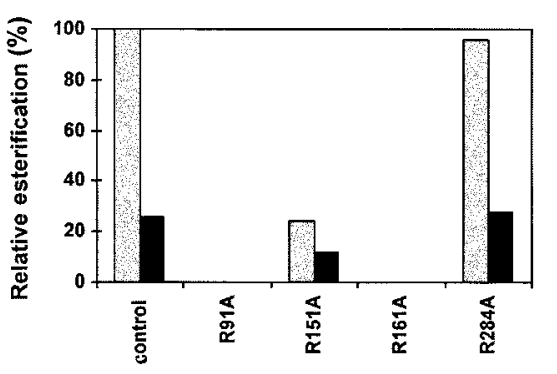

(B)

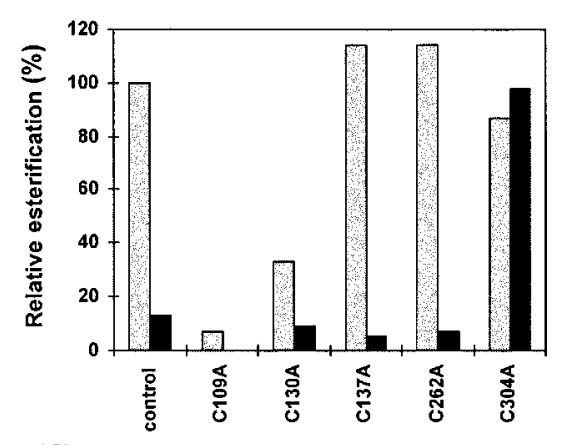

(C)

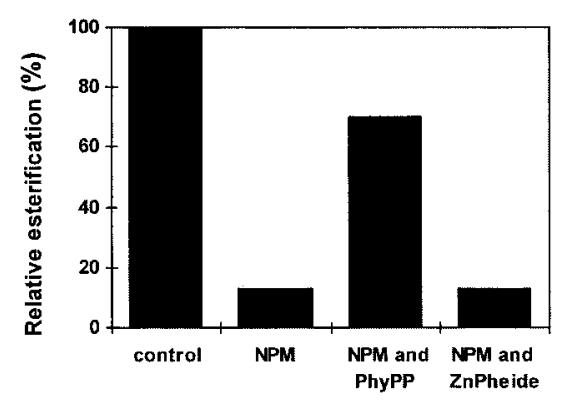

Fig. 6 Activity of Mutated Chlorophyll Synthases with and without Inhibitors.

(A) Arginine residues of the core region were mutated to alanin, and the resulting mutated enzymes were tested in the absence (grey) and presence (black) of $34 \mathrm{~mm}$ diacetyl.

(B) Cysteine residues were mutated to alanine, and the resulting mutated enzymes were tested in the absence (grey) and presence (black) of $4 \mathrm{~mm} \mathrm{~N}$-phenylmaleimide.

(C) The recombinant enzyme (wild type) was preincubated for 30 min with either NPM, $360 \mu \mathrm{M}$ PhyPP and NPM, or $6 \mu \mathrm{M}$ Zn Pheide and NPM. Only PhyPP abolished inhibition by NPM.

zymes was tested without and with NPM. As shown in Figure $6 \mathrm{~B}$, only some of the results matched the expectation. The mutant C109A, in which the only conserved Cys residue was substituted by Ala, exhibited nearly no enzyme activity. Nevertheless, NPM treatment resulted in an additional decrease of activity. The mutant C130A showed reduced activity and sensitivity to NPM, the mutants C137A and C262A were not impaired in their enzyme activity and showed the same inhibition by NPM as the wild-type. Surprisingly, NPM did not inhibit the activity of the mutant C304A. The activity with or without NPM was as high as that of the wild-type control without NPM. This finding leads to two conclusions: the thiol-group of Cys-304 is not essential for the reaction since the activity 
of the mutant C304A is as high as that of the wild type. The presence of the thiol-group of Cys-304 is the precondition for inhibition of the enzyme activity by NPM. To test whether the inhibition by NPM involved specifically the binding of one of the substrates, the inhibitor was appplied together with saturating concentrations of either substrate (Figure 6C). Zn Pheide did not protect the enzyme against inhibition by NPM, but PhyPP was able to reduce the inhibition.

\section{Discussion}

Gene sequences and the derived amino acid sequences of bacteriochlorophyll synthases and chlorophyll synthases have so far been described for several phototrophic bacteria and the dicotyledonous plant Arabidopsis thaliana (see Table 1). We describe here the first chlorophyll synthase sequence from a monocotyledonous plant. The high degree of similarity between such distant organisms as Avena sativa and Synechocystis (62\% identity) indicates that there must have been restrictions to sequence variation during evolution. Such restrictions can indicate that the particular sequence is required for the functionality of the enzyme. Binding of two different amphiphilic substrates (chlorophyllide and PhyPP), probably entering the active site from the stromal side, and their reaction to the lipophilic product $\mathrm{Chl}$, which is probably released to the membrane phase, may require a well-defined spatial structure, which in turn depends on the amino acid sequence.

Nine membrane-spanning helices were predicted for chlorophyll $a$ and bacteriochlorophyll synthases using the program HMMTOP. The predicted structure seems to be similar to the 'terpenoid synthase fold', normally forming a large central cavity by 10 helices containing the conserved aspartate-rich motifs (Wang and Ohnuma, 1999). Using the program PEPPLOT, Lopez et al. (1996) predicted 8 membrane-spanning helices for bacteriochlorophyll synthase from Chloroflexus aurantiacus. Their program predicts only 6 membrane-spanning helices for chlorophyll synthases. Taking into account the high degree of similarity in the amino acid sequences and in the enzymatic reaction, we consider such a large difference in the secondary structure to be unlikely.

The failure to find a second chlorophyll synthase gene prompted us to reinvestigate the substrate binding specificity with GGPP and PhyPP. In etioplasts and chloroplasts, PhyPP and GGPP gave indistinguishable results when they were applied at saturating concentration and when only the end point of the chlorophyll synthase reaction was analysed. Differences were found when either both substrates were mixed and reacted in competition (Rüdiger et al., 1980) or when the kinetics of the reaction was analysed (Soll et al., 1983). Under both conditions, the recombinant enzyme from $A$. sativa reacts faster with GGPP than with PhyPP at the beginning, indicating a preference for GGPP at short reaction periods. In con- trast, the final product yield is higher with PhyPP than with GGPP, indicating a preference for PhyPP if only long reaction periods are analysed. Since the reaction time varies with the substrate:enzyme ratio, such variation of the reaction conditions can simulate different 'preferences' with one and the same enzyme. Thus the apparent differences found with the substrates PhyPP and GGPP cannot be taken as a criterion for the existence of different chlorophyll synthases.

Protein prenylation by farnesyl or geranylgeranyl residues is well known (Gelb et al., 1998). Several functions have been assigned to prenylated proteins, ranging from fungal mating factors and mammalian lamin which is essential for the structure of the nuclear envelope, to Ras and Rab proteins which are involved in intracellular signaling and vesicle trafficking, respectively. The site of prenylation is a Cys residue at or close to the C-terminus in most of these proteins. There is no Cys residue close to the C-terminus in chlorophyll synthase. If Cys is considered as a possible binding site for the phytyl or geranylgeranyl residue in chlorophyll synthase, it could only be the Cys at position 109. This Cys is conserved in all known chlorophyll and bacteriochlorophyll synthases, and the mutant C109A was nearly devoid of activity. It should be kept in mind, however, that the function of chlorophyll synthase is the transfer of the polyprenyl residue to the second substrate Chlide, while the hitherto described prenyl proteins are permanently modified at the Cys residue. However, we cannot exclude the possibility that chlorophyll synthases react not only with the polyprenyl residue to be transferred to Chlide but also with an additional polyprenyl residue under permanent modification, and that this modification is necessary for activity. An alternative function for the essential Cys-109 of chlorophyll synthase could be the stabilization of the active conformation of the enzyme. Most likely, such stabilization does not occur via a disulfide bridge in this case because the enzyme does not contain the counterpart, i. e. a second essential Cys residue. Interestingly, inhibition of the enzyme activity by NPM occurs via reaction with Cys-304 which is not essential. Since only PhyPP (and not Zn Pheide) counteracts the inhibition, we conclude that reaction of Cys-304 with the bulky NPM leads to a steric hindrance of PhyPP binding.

Lopez et al. (1996) pointed out that bacteriochlorophyll and chlorophyll synthases belong to the family of polyprenyltransferases and that a homologous region ('domain II') has been suggested to be the binding site of the polyprenyl PP. In the sequence of $A$. sativa, this region includes the residues 138 to 160 . Two possibilities have been discussed for interaction of the negatively charged phosphates of polyprenyl diphosphates with the active center of polyprenyl transferases, either $R$ and $K$ residues as counterions (Kral et al., 1997) or acidic groups of a DDXXD motif via complexed $\mathrm{Mg}^{2+}$ ions (Tarshis et al., 1996; Wang and Ohnuma, 1999). Indirect evidence presented in this paper shows that both possibilities apply also for chlorophyll synthase. 
The similar motif DRXXD occurs in the domain II homolog of bacteriochlorophyll synthase (Lopez et al., 1996). The alignment (Figure 1) reveals that the same motif is present in chlorophyll synthase from $A$. sativa starting with D150, and an overlapping motif NDXXD within the same domain starting with N146. Lopez et al. (1996) stated correctly that the requirement of chlorophyll synthases for $\mathrm{Mg}^{2+}$ or other divalent cations has not explicitly been investigated. We found now that the enzyme from A. sativa requires $\mathrm{Mg}^{2+}$ ions, which cannot be substituted by other divalent ions for full activity.

Point mutations (see Figure 6A) revealed essential Arg residues also in chlorophyll synthase. Interactions of Arg and Lys residues with the phosphate groups of farnesyldiphosphate were shown by X-ray analysis of the crystal structure and by site-directed mutagenesis of human farnesyl: protein transferase (Kral et al., 1997; Park et al., 1997, Long et al., 1998). On the basis of the crystal structure of farnesyldiphosphate synthase, two Arg residues were discussed either for binding the allylic diphosphate during catalysis or for pulling the diphosphate residue after lysis out of the active site (Tarshis et al., 1996). In the absence of precise structural information, we can only speculate about the function of essential Arg residues in chlorophyll synthase. Arg-161, one of the two essential Arg residues of chlorophyll synthase, is located directly at the end of the presumed prenyl binding site and is thus a candidate for the correct binding of PhyPP or GGPP. The other essential residue, Arg-91, could well be important for interaction with the diphosphate after lysis, but other functions cannot be excluded at this time.

\section{Materials and Methods}

\section{Plant Material}

Seedlings of Avena sativa L. cv Pirol (Baywa, München, Germany) were grown on moist vermiculite at $23^{\circ} \mathrm{C}$ either in a dark room or under continuous white light $\left(32 \mathrm{~W} \mathrm{~m}^{-2}\right)$ for 3,4 , and 5 days. Some batches of seedlings were harvested directly. Other batches of the dark-grown seedlings were then placed under white light for the indicated period of time $(0.5,1,2,4,8 \mathrm{~h})$ before harvest. The coleoptiles were removed, the primary leaves (100-800 mg fresh weight per batch) were cut, immediately frozen in liquid nitrogen, and stored at $-80^{\circ} \mathrm{C}$ until use. Etioplasts were prepared from 8 day old etiolated oat seedlings according to Klement et al. (1999). The plastids were broken through several freezing-thawing cycles. For the enzyme assay $1 \mathrm{mg}$ of total protein was taken.

\section{Cloning of the Chlorophyll Synthase Gene}

Double-stranded cDNA was prepared according to Sambrook et al. (1989) from poly(A)-RNA which was isolated from $4 \mathrm{~d}$ old etiolated oat seedlings. With the forward primer F1 (5'-GGCCTCCA(CT)T(ACTG)GTTTGGGG-3) and the reverse primer R1 (5'CCAAGAATGGCTGCGCGCTTGCCTG-3), a 754 bp fragment of the coding region of chlorophyll synthase was obtained. The missing parts upstream and downstream of this fragment were then prepared with the RACE-PCR method (Frohmann et al., 1988; Rashtchian et al., 1992) using sequence specific primers as follows: for the upstream part the forward primers F2 (5'-GGCCACGCGTCGACTAGTACGGG॥GGG॥GGGIIG-3) and F3 (5'-GGCCACGCGTCGACTAGTAC-3) and the reverse primers R2 (558-581, 5'-CATATGTCTAACAAAGCACCCAAC-3) and R3 (534-557, 5'-CCAAGACCTCCTAACAATAATACC-3), for the downstream part the forward primers F4 (558-581, 5'-GTTGGGTGCTTTGTTAGACATATG-3) and F5 (583-599, 5'-GCAGGGCATGATTTTCC-3) and the reverse primers R4 $\left(5^{\prime}\right.$-GGCCACGCGTCGACTAGTAC $\left(\mathrm{T}_{17} \mathrm{1}^{-} \mathbf{3}^{\prime}\right)$ and R5 (5'-GGCCACGCGTCGACTAGTAC-3). All PCRs were performed with the following program: initial melting at $94^{\circ} \mathrm{C}$ for $5 \mathrm{~min}$, then $30 \mathrm{cy}$ cles of 1 min melting at $92^{\circ} \mathrm{C}, 1$ min annealing (temperature depending on the melting temperature of the primer pair) and 2-4 min elongation. All fragments were cloned into the pCR-Script vector (Stratagene, La Jolla, USA) and sequenced using oligonucleotides priming in the T3/T7 promotor region. The fulllength sequence (1428 bp) was then obtained by PCR with the sequence-specific primers F6 (5'-TAGGTACCATGGCCACCTCCCACCT-3), which introduces an Ncol restriction site, and R6 (5'-CGCATAAGCTTCAAGGGGAAACAATTTG-3), which introduces a TGA stop codon and a Hindlll restriction site. For the sequence from green plants, the primer R7 (5'-CGCATAAGCTCCAATATCGGAACAATC-3) was used instead of R6. The resulting PCR fragment was cut with $\mathrm{NcOl}$ and Hindlll and ligated to the vector PQE-60 (Qiagen, Hilden, Germany) which had been restricted before with the same enzymes.

\section{RNA Blotting and Hybridization}

For Northern analysis, a probe was prepared from the $754 \mathrm{bp}$ fragment, cloned into the pCR-Script vector, by restriction with $\mathrm{BamHI}$, resulting in a fragment of $450 \mathrm{bp}$. This fragment was labelled with $\left.{ }^{32} \mathrm{P}\right]$ by random priming (Feinberg and Vogelstein, 1983).

Total RNA was isolated as described by Paulsen and Bogorad (1988) with the modifications introduced by Kittsteiner et al. (1991). Samples containing the equal amounts of RNA were subjected to electrophoresis and transferred to nitrocellulose. For quantitation of the levels of chlorophyll synthase mRNA, aliquots of the RNA solution and 1:3 dilutions were loaded into a slot blot apparatus (Minifold II, Schleicher and Schuell). Washing, prehybridisation and hybridisation were performed as described by Paulsen and Bogorad (1988).

\section{In Vitro Mutagenesis}

Mutagenesis was performed according to the 'quickchange' site-directed mutagenesis protocol (Stratagene, La Jolla, USA) using Pwo polymerases (Peqlab, Erlangen, Germany) and the restriction enzyme Dpnl (NEB, Schwalbach). The mutated plasmids were transformed into E. coli XL1 Blue cells by electroporation (EasyJect Prima, Peqlab). The transformants were grown on LB medium containing ampicillin $(100 \mu \mathrm{g} / \mathrm{ml})$. After verification by restriction analysis and sequencing, the mutated plasmids were transformed into cells of the E. coli strain SG13009 (Qiagen), and the transformants were grown on agar plates containing $2.5 \%$ trypton/pepton, $1.5 \%$ yeast extract, $0.6 \% \mathrm{NaCl}$, ampicillin $(100 \mu \mathrm{g} / \mathrm{ml})$ and kanamycin $(25 \mu \mathrm{g} / \mathrm{ml})$. The expression was performed at room temperature. At an $\mathrm{OD}_{600}=0.6$ protein expression was induced by $0.3 \mathrm{~mm} \mathrm{IPTG}$ and were grown for another $4 \mathrm{~h}$.

\section{Enzyme Assay}

The induced bacterial cells (containing 250-300 mg of total protein per batch grown in $500 \mathrm{ml}$ of medium) were harvested by centrifugation (6000 $\mathrm{g}$ for $15 \mathrm{~min}$ ), washed with $15 \mathrm{ml} 50 \mathrm{~mm}$ 
HEPES ( $\mathrm{pH} 8.0)$, and broken after adding lysozyme $(300 \mu \mathrm{g})$ and DNase I $(300 \mu \mathrm{g})$ (final volume $3 \mathrm{ml}$ ) by several cycles of freezing and thawing. Aliquots of the suspension containing $3 \mathrm{mg}$ total protein were used directly for the enzyme reaction in most experiments. In some experiments, the suspension was preincubated with one of the inhibitors diacetyl or N-phenylmaleimide for $30 \mathrm{~min}$ at room temperature. To investigate the role of $\mathrm{Mg}^{2+}$ for the enzymatic reaction, $\mathrm{Mg}^{2+}$ was removed. In this case, the cell fragments were pelleted at $16000 \mathrm{~g}$ and incubated with 50 mM HEPES ( $\mathrm{pH}$ 8.0) containing $100 \mathrm{~mm}$ EDTA for 30 min, pelleted again and washed 4 times with $50 \mathrm{mM}$ HEPES (pH 8.0). The EDTA-treated membrane samples were then incubated for 30 min with $4 \mathrm{~mm}$ of various salts $\left(\mathrm{MgCl}_{2}, \mathrm{MnCl}_{2}, \mathrm{ZnCl}_{2}, \mathrm{CaCl}_{2}\right)$ in 50 mM HEPES (pH 8.0) and subsequently used for the enzyme test (see Table 2).

Aliquots of the bacterial lysate or the pretreated membranes (containing $3 \mathrm{mg}$ of total protein) or the broken etioplasts were mixed with $90 \mathrm{nmol}$ GGPP and 50 mM HEPES (pH 8.0) to a total volume of $240 \mu \mathrm{l}$. The reaction was started under dim-green safelight by addition of $1 \mathrm{nmol}$ chlorophyllide a (Chlide) (if not stated otherwise) dissolved in $10 \mu$ acetone. After incubation for $45 \mathrm{~min}$ at $29^{\circ} \mathrm{C}$, the reaction was stopped by addition of $750 \mu \mathrm{l}$ acetone, and the precipitated protein was removed through centrifugation. After addition of $40 \mu \mathrm{l} 100 \mathrm{mM} \mathrm{Na}_{2} \mathrm{CO}_{3}$, the clear supernatant was used to quantitate the esterified $\mathrm{Chl}$ by phase separation as previously described (Helfrich et al., 1994).

\section{Acknowledgements}

We thank Mr. A. Beck for preliminary experiments with inhibitors and Dr. S. Beale for helpful discussions. The work was supported by the Deutsche Forschungsgemeinschaft, Bonn (SFB 184) and the Fond der Chemischen Industrie, Frankfurt, Germany.

\section{References}

Barnard, G., and Popjak, G. (1980). Characterization of liver prenyl transferase and its inactivation by phenylglyoxal. Biochim. Biophys. Acta 617, 169-182.

Bennett, J. (1981). Biosynthesis of the light-harvesting chlorophyll $\mathrm{a} / \mathrm{b}$ protein. Polypeptide turnover in darkness. Eur. J. Biochem. 118, 61-70.

Benz, J., and Rüdiger, W. (1981a). Chlorophyll biosynthesis: various chlorophyllides as exogenous substrates for chlorophyll synthetase. Z. Naturforsch. 36c, 51-57.

Benz, J., and Rüdiger, W. (1981b). Incorporation of 1-14Cisopentenyldiphosphate, geraniol and farnesol into chlorophyll in plastid membrane fractions of Avena sativa L. Z . Pflanzenphysiol. 102, 95-100.

Benz, J., Wolf, C., and Rüdiger, W. (1980). Chlorophyll biosynthesis: hydrogenation of Geranylgeranol. Plant Sci. Lett. 19 225-230.

Benz, J., Hampp, R., and Rüdiger, W. (1981). Chlorophyll biosynthesis by mesophyll protoplasts and plastids from etiolated oat (Avena sativa L.) leaves. Planta 152, 54-58.

Benz, J., Haser, A., and Rüdiger, W. (1983). Changes in the endogenous pools of tetraprenyl diphosphates in etiolated oat seedlings after irradiation. Z. Pflanzenphysiol. 111, 349-356.

Eichacker, L. A., Soll, J., Lauterbach, P., Rüdiger, W., Klein, R. R., and Mullet, J. E. (1990). In vitro synthesis of chlorophyll a in the dark triggers accumulation of chlorophyll a apoproteins in barley etioplasts. J. Biol. Chem. 265, 13566-13571.

Eichacker, L., Paulsen, H., and Rüdiger, W. (1992). Synthesis of chlorophyll a regulates translation of chlorophyll a apopro- teins P700, CP47, CP43 and D2 in barley etioplasts Eur. J. Biochem. 205, 17-24.

Eichacker, L. A., Helfrich, M., Rüdiger, W., and Müller, B. (1996). Stabilization of chlorophyll a-binding apoproteins P700, CP47, CP43, D2, and D1 by chlorophyll a or Zn-pheophytin a. J. Biol. Chem. 271, 32174-32179.

Emanuelsson, O., Nielsen, H., and von Heijne, G. (1999). ChloroP, a neural network-based method for predicting chloroplast transit peptides and their cleavage sites. Protein Sci. 8, 978-984.

Feinberg, A. P., and Vogelstein, B. (1983). A technique for radiolabeling DNA restriction endonuclease fragments to high specific activity. Anal. Biochem. 132, 6-13.

Frohmann, M. A., Dush, M. K., and Martin, G. R. (1988). Rapid production of full-length cDNAs from rare transcripts: Amplification using a single gene-specific oligo nucleotide primer. Proc. Natl. Acad. Sci. USA 85, 8998-9002.

Gaubier, P., Wu, H.-J., Laudie, M., Delseny, M., and Grellet, F. (1995). A Chl synthetase gene from Arabidopsis thaliana. Mol. Gen. Genet. 249, 58-64.

Gavel, Y., and von Heijne, G. (1990). A conserved cleavage-site motif in chloroplast transit peptides. FEBS Lett. 261, 455-458.

Gelb, M. H., Scholten, J. D., and Sebolt-Leopold, J. S.(1998). Protein prenylation: from discovery to prospects for cancer treatment. Curr. Opin. Chem. Biol. 2, 40-48.

Helfrich, M., and Rüdiger, W. (1992). Various metallopheophorbides as substrates for chlorophyll synthetase. Z. Naturforsch. 47 c, 231-238.

Helfrich, M., Schoch, S., Lempert, U., Cmiel, E., and Rüdiger, W. (1994). Chlorophyll synthetase cannot synthesize chlorophyll a'. Eur. J. Biochem. 219, 267-275.

Keegstra, K., and von Heijne, G. (1992). Transport of proteins into chloroplasts. In: Plant Gene Research: Cell Organelles, R.G. Herrmann, ed. (Berlin, Germany: Springer), pp. 353-370.

Kim, J., Eichacker, L. A., Rüdiger, W., and Mullet, J. E. (1994). Chlorophyll regulates accumulation of the plastid-encoded chlorophyll proteins P700 and D1 by increasing apoprotein stability. Plant Physiol. 104, 907-916.

Kittsteiner, U., Brunner, H., and Rüdiger, W. (1991). The greening process in cress seedlings. II. Complexing agents and 5aminolevulinate inhibit accumulation of cab-mRNA coding for the light-harvesting chlorophyll a/b protein. Physiol. Plant. 81, 190-196.

Klein, R. R., Gamble, P. E., and Mullet, J. E. (1988). Regulation of chlorophyll a-apoprotein accumulation. Plant Physiol. 88, $1246-1256$.

Klement, H., Helfrich, M., Schoch, S., and Rüdiger, W. (1999). Pigment-free NADPH:protochlorophyllide oxidoreductase from Avena sativa $L$ : purification and substrate specificity. Eur. J. Biochem. 265, 862-874.

Kral, A. M., Diehl, R. E., deSolms, S. J., Williams, T. M., Kohl, N. E., and Omer, C. A. (1997). Mutational analysis of conserved residues of the $B$-subunit of human farnesyl:protein transferase. J. Biol. Chem. 272, 27319-27323.

Long, S. B., Casey, P.J., and Beese, L.S. (1998). Cocrystal structure of protein farnesyltransferase complexed with a farnesyl diphosphate substrate. Biochemistry 37, 9612-9618.

Lopez, J. C., Ryan, S., and Blankenship, R. E. (1996). Sequence of the bxhG gene from Chloroflexus aurantiacus: relationship between chlorophyll synthase and other polyprenyltransferases. J. Bacteriol. 178, 3369-3373.

Lütz, C., Benz, J., Rüdiger, W., and Eder, J. (1981). Esterification of chlorophyllide in prolamellar body (PLB) and prothylakoid (PT) fractions from Avena sativa etioplasts. Z. Naturforsch. $36 c, 58-61$. 
Oster, U., and Rüdiger, W. (1997). The G4 gene of Arabidopsis thaliana encodes a chlorophyll synthase of etiolated plants. Bot. Acta 110, 420-423.

Oster, U., Bauer, C. E., and Rüdiger, W. (1997). Characterization of chlorophyll a and bacteriochlorophyll a synthases by heterologous expression in Escherichia coli. J. Biol. Chem. 272, 9671-9676.

Park, H. W., Boduluri, S. R., Moonmaw, J. F., Casey, P. J., and Beese, L. S. (1997). Crystal structure of protein farnesyltransferase at 2.25 Å resolution. Science 275, 1800-1804.

Paulsen, H., and Bogorad, L. (1988). Diurnal and circadian rhythms in the accumulation and synthesis of mRNA for the light-harvesting chlorophyll a/b-binding protein in tobacco. Plant Physiol. 88, 1104-1109.

Rashtchian, A., Buchman, G. W., Schuster, D. M., and Berninger, M. S. (1992). Uracil DNA glycosylase-mediated cloning of polymerase chain reaction-amplified DNA: application to genomic and cDNA cloning. Anal. Biochem. 206, 91-97.

Rüdiger, W., Benz, J., and Guthoff, C. (1980). Detection and partial characterization of activity of chlorophyll synthetase in etioplast membranes. Eur. J. Biochem. 109, 193-200.

Sambrook, J., Fritsch, E. F., and Maniatis, T. (1989). Molecular
Cloning: A Laboratory Manual, $2^{\text {nd }}$ Edition (Cold Spring Harbor, New York: Cold Spring Harbor Laboratory Press).

Schoch, S., Hehlein, C., and Rüdiger, W. (1980). Influence of anaerobiosis on chlorophyll biosynthesis in greening oat seedlings (Avena sativa L.). Plant Physiol. 66, 576-579.

Soll, J., Schultz, G., Rüdiger, W., and Benz, J. (1983). Hydrogenation of geranylgeraniol: two pathways exist in spinach chloroplasts. Plant Physiol. 71, 849-854.

Tarshis, L. C., Proteau, P. J., Kellogg, B. A., Sacchettini, J. C., and Poulter, C. D. (1996). Regulation of product chain length by isoprenyl diphosphate synthases. Proc. Natl. Acad. Sci. USA 93, 15018-15023.

von Heijne, G., and Nishikawa, K. (1991). Chloroplast transit peptides. The perfect random coil? FEBS Lett. 278, 1-3.

von Heijne, G., Steppuhn, J., and Herrmann, R.G. (1989). Domain structure of mitochondrial and chloroplast-targeting peptides. Eur. J. Biochem. 180, 535-545.

Wang, K., and Ohnuma, S. (1999). Chain-length determination mechanism of isoprenyl diphosphate synthases and implications for molecular evolution. Trends Biochem. 24, 445-451.

Received December 19, 2000; accepted February 20, 2001 